\title{
CD99 activates T cells via a costimulatory function that promotes raft association of TCR complex and tyrosine phosphorylation of TCR $\zeta$
}

\author{
Kwon Ik Oh${ }^{1 *}$, Byoung Kwon $\mathrm{Kim}^{2 *}$, \\ Young Larn $\mathrm{Ban}^{3}$, Eun Young $\mathrm{Choi}^{3,4}$, \\ Kyeong Cheon Jung ${ }^{2,3}$, Im-Soon Lee $^{5}$ and \\ Seong Hoe Park ${ }^{2,3,4,6}$ \\ 1Department of Pathology \\ Hallym University College of Medicine \\ Chuncheon 200-702, Korea \\ ${ }^{2}$ Department of Pathology \\ ${ }^{3}$ Graduate Program of Immunology \\ ${ }^{4}$ Center for Animal Resource Development \\ Seoul National University College of Medicine \\ Seoul 110-799, Korea \\ ${ }^{5}$ Department of Biological Sciences \\ College of Sciences Konkuk University \\ Seoul 143-701, Korea \\ ${ }^{6}$ Corresponding author: Tel, 82-2-740-8264; \\ Fax, 82-2-763-6625; E-mail, pshoe@plaza.snu.ac.kr \\ *These authors contributed equally to this work.
}

Accepted 7 February 2007

Abbreviations: TCR, T-cell receptor

\begin{abstract}
We investigated the co-stimulatory role of a cellsurface protein, CD99. Co-ligation of CD99 and suboptimal CD3 induced T-cell activation to a level comparable to that obtained with optimal CD3 or CD3+CD28. We also noted concomitant enhancement of the earliest T-cell receptor (TCR) signaling events. In addition, co-ligation of CD99 and CD3 led to translocation of TCR complexes into the lipid raft, without concomitant migration of CD99 to the raft, and consequent enhancement of TCR $\zeta$-mediated signal 1. These data demonstrate the unique properties of CD99 co-stimulation that distinguish this molecule from CD28 and other raft-resident costimulatory factors.
\end{abstract}

Keywords: antigens; lymphocyte activation; receptors, antigen, T-cell; signal transduction; T lymphocytes

\section{Introduction}

The physiological process of T-cell activation can be divided into a series of temporal stages consisting of initial adhesion, initial signaling (formation of the contact cap), and sustained signaling (Bromley et al., 2001). During these stages, two independent signals are required for the enactment of full T-cell activation: (1) specific interaction of a T-cell receptor (TCR) with a cognate peptide/MHC on APCs, and (2) the ligation of surface co-stimulatory molecules with specific ligands on APCs. Among the known costimulatory molecules, CD28 launches the most effective activation of $\mathrm{T}$ cells (Alegre et al., 2001; Frauwirth and Thompson, 2002). However, recent reports have suggested that many other candidates, most of which reside in the lipid raft, induce T-cell activation as potently as CD28 does (Denning et al., 1988; Ledbetter et al., 1988; Green et al., 1994; Tai et al., 1996; Yashiro-Ohtani et al., 2000; Stillwell and Bierer, 2001).

The lipid raft is a unique membrane microdomain that is enriched with specific types of lipids and cholesterol and is associated with many key molecules involved in TCR signaling. Upon engagement of cognate ligands (peptide/MHC), TCRs are recruited toward the raft along with a series of tyrosine-phosphorylated signaling molecules (Moran and Miceli, 1998; Xavier et al., 1998; Janes et al., 1999). The lipid raft thus serves as a stage for protein-protein interactions among many surface molecules, thereby instigating a series of signaling cascades following TCR engagement (Cherukuri et al., 2001).

CD99, a monomeric 32-kDa transmembrane glycoprotein, is the product of mic2 and lacks significant homology to any functional or structural domains of known proteins (Schenkel et al., 2002). Ligation of CD99 with anti-CD99 mAb induces homotypic aggregation and apoptosis of thymocytes (Bernard et al., 1997) as well as up-regulation of cell-surface proteins, such as TCR and MHC classes I and II (Choi et al., 1998). A recent report suggested that reorganization of the cytoskeleton is involved in the function of CD99 (Cerisano et al., 2004).

In this study, we explored the possible role and mechanism of CD99 as a co-stimulatory molecule in T-cell activation. CD99 engagement leads to the 
recruitment of TCR/CD3 into lipid rafts. However, CD99 enhances T-cell activation through increasing signal 1 , such as phosphorylation of TCR $\zeta$, only when a suboptimal level of signal is provided by TCR/CD3 ligation. Our results indicate another role of CD99 in T cells, i.e., co-stimulation, the underlying mechanism of which differs in many ways from those of other co-stimulatory molecules involved in T-cell activation.

\section{Materials and Methods}

\section{Cell culture}

Jurkat T cells (clone E6-1, obtained from American Type Culture Collection; Rockville, MD) were maintained in culture in RPMI-1640 supplemented with $5 \%$ FCS, 2 mM L-glutamine, $0.1 \%$ penicillin/streptomycin. Human PBMC were isolated from heparinized blood of healthy adult donors by standard density gradient centrifugation with Ficoll-Paque (Amersham Pharmacia; Uppsala, Sweden). Isolated PBMC were maintained in culture in RPMI-1640 supplemented with $10 \%$ autologous serum.

\section{Antibodies}

The following mAbs were used in this study: antiCD99 (DiNonA; Seoul, Korea), biotinylated or FITC labeled anti-CD3 (UCHT1, DiNonA), goat or rabbit anti-mouse secondary antibodies (DiNonA), antiCD3 and CD1a (OKT3 and OKT6 respectively for cell stimulation; American Type Culture Collection), anti-CD3 \& (M-20 for immunoblotting, Santa Cruz Biotechnology; Santa Cruz, CA), anti-CD3 $\zeta$ (6B10.2, Santa Cruz Biotechnology), anti-phosphotyrosine (4G10, Upstate Biotechnology; Lake Placid, NY), anti-transferrin receptor (16E, a generous gift from Junji Kato, Fourth Department of Internal Medicine, Sapporo Medical University School of Medicine, Sapporo, Japan), anti-Ick (3A5, Santa Cruz Biotechnology) and anti-CD28 (CD28.2, BD Pharmingen; San Diego, CA ).

\section{Proliferation assays}

Proliferation assays were performed using standard techniques (Waclavicek et al., 1998). Briefly, $10^{5}$ PBMC/well were cultured for $72 \mathrm{~h}$ in mAb-coated 96-well plates in RPMI media supplemented with autologous sera, and pulsed with a $\left[{ }^{3} \mathrm{H}\right]$ thymidine solution ( $1 \mu \mathrm{Ci} /$ well, $6.7 \mathrm{Ci} / \mathrm{mmol}$ specific activity, Amersham; Aylesbury, UK) for the last $18 \mathrm{~h}$ before harvesting. The mean incorporation of thymidine in DNA was measured in quadruplicate wells by liquid scintillation counting.

\section{TCR down-regulation}

PBMC or Jurkat cells were incubated at $37^{\circ} \mathrm{C}$ in wells previously coated with desired mAbs for the indicated time (Kim et al., 2004; Zhang et al., 2005). The cells then were resuspended, washed and stained with the specific Abs directly labeled with FITC or PE. The mean fluorescence intensity of each sample was measured at each point by FACScalibur flow cytometer (BD Bioscience; San Jose, CA).

\section{Isolation of lipid rafts}

Lipid rafts were isolated using modified lysis conditions and flotation on discontinuous sucrose gradients (Montixi et al., 1998). Briefly, Jurkat T cells were lysed and the lysed solution was further homogenized with 10 strokes in a Wheaton loosefitting dounce homogenizer. Nuclei and cellular debris were pelleted and the supernatant was adjusted to $1.33 \mathrm{M}$ sucrose by adding $2 \mathrm{ml}$ of $2 \mathrm{M}$ sucrose prepared in buffer TNEV $(50 \mathrm{mM}$ TrisCl, $\mathrm{pH} 7.5,150$ $\mathrm{mM} \mathrm{NaCl}, 5 \mathrm{mM}$ EDTA, and $1 \mathrm{mM} \mathrm{Na}_{3} \mathrm{VO}_{4}$ ), placed at the bottom of an ultracentrifuge tube, and overlayed with a step sucrose gradient $(0.2-0.9 \mathrm{M}$ with $0.1 \mathrm{M}$ steps, $1 \mathrm{ml}$ each) in buffer TNEV. The tube was centrifuged at 38,000 r.p.m. for $16 \mathrm{~h}$ in an SW41 rotor (Beckman Instruments Inc; Palo Alto, CA) at $3^{\circ} \mathrm{C}$, and $1 \mathrm{ml}$ fractions were harvested from the top.

\section{Immunoprecipitation, and Western blot analysis}

For immunoprecipitations (Jang et al., 2004), total cell lysates were pre-cleared and then incubated overnight at $4{ }^{\circ} \mathrm{C}$ with antibody coupled to protein $\mathrm{A}$ beads (Pierce; Rockford, IL). Protein samples were resolved on $12 \%$ SDS-PAGE and visualized using ECL kit (Amersham; Little Chalfont, Buckinghamshire, UK).

\section{Immunofluorescence microscopy}

Jurkat $\mathrm{T}$ cells were stimulated with $\mathrm{mAb}$-coated beads on the slides, and rinsed in PBS and fixed in $4 \%$ paraformaldehyde for $20 \mathrm{~min}$. After quenching paraformaldehyde with $50 \mathrm{mM} \mathrm{NH}_{4} \mathrm{Cl}$, the cells were permeabilized in $0.1 \%$ saponin for $10 \mathrm{~min}$, and blocked with a solution of $0.01 \%$ saponin and $1 \%$ BSA. Cholera toxin-B-FITC/biotin, anti-CD3-biotin and antiCD99-FITC were used to label the conjugates.

\section{Results}

\section{Enhanced activation of $\mathrm{T}$ cells by co-ligation of CD99 and CD3}

The co-stimulatory function of CD99 in T-cell acti- 
vation was demonstrated by a $\left[{ }^{3} \mathrm{H}\right]$-thymidine incorporation assay using PBMC cultured in the presence of anti-CD99 mAb (DN16) plus various concentrations of anti-CD3 mAb (OKT3). Co-ligation of CD99 and CD3 markedly enhanced cell activation, allowing treated cells to proliferate and secret IL-2 at suboptimal concentrations of anti-CD3 (Figure 1A and $\mathrm{B}$ ). Enhanced cell activation by co-ligation of CD99 was also evident from the increased surface expression of the activation marker CD69 and the
A

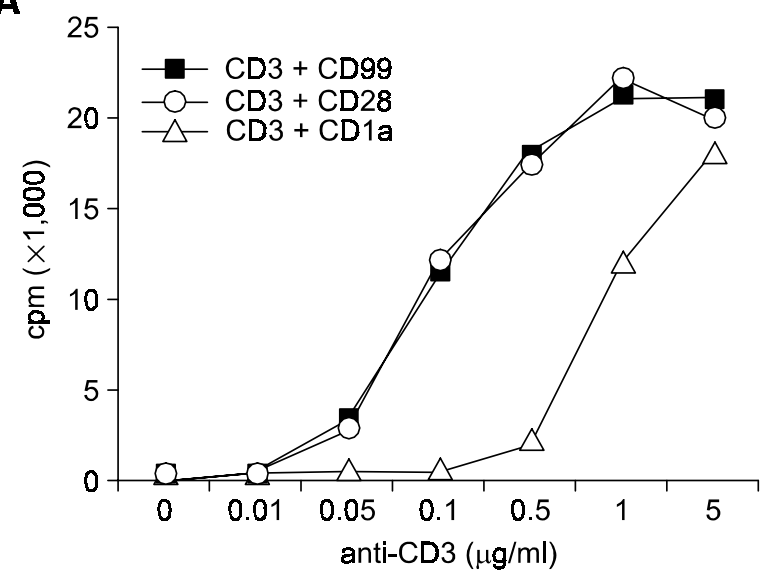

B

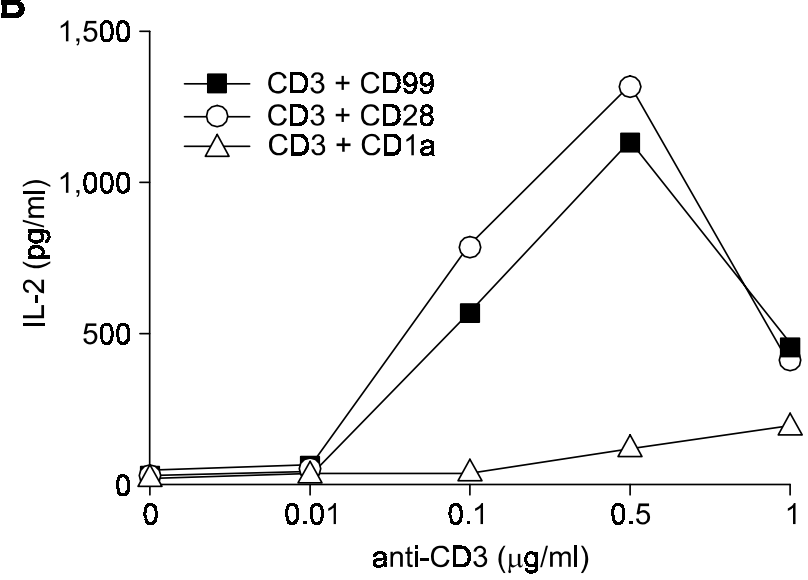

C

anti-CD3 $(0 \mu \mathrm{g} / \mathrm{ml})$

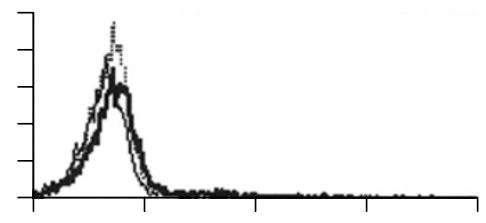

$\mathrm{CD} 3+\mathrm{CD} 1 \mathrm{a}$

D

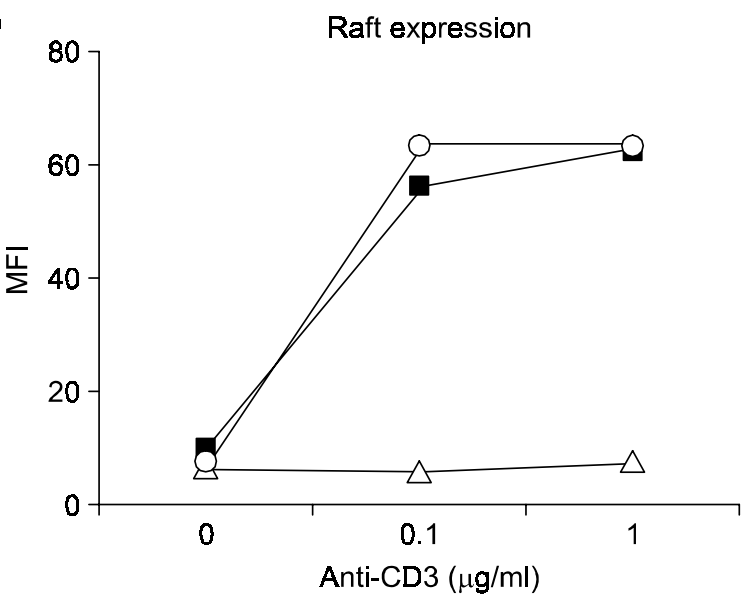

anti-CD3 $(0.1 \mu \mathrm{g} / \mathrm{ml})$

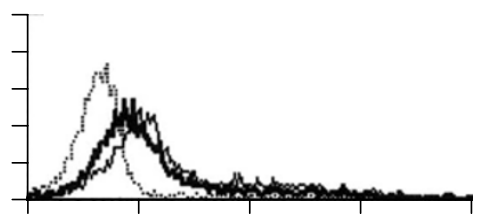

CTX-FITC

$\mathrm{CD} 3+\mathrm{CD} 28$
anti-CD3 $(1 \mu \mathrm{g} / \mathrm{ml})$
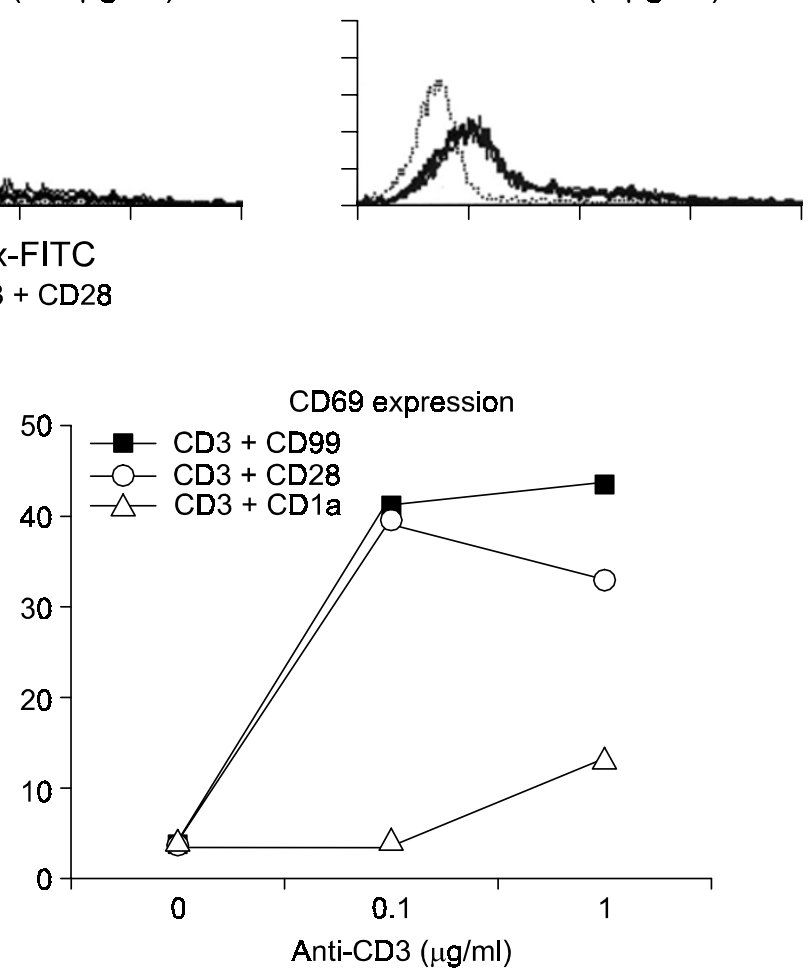

Figure 1. CD99 engagement facilitates a T-cell response in the presence of suboptimal CD3 stimulation. (A, B) Human PBMC were stimulated in 96-well plates with anti-CD3 together with anti-CD99, CD28, or CD1a (an isotype-matched control) for $72 \mathrm{~h}$. (C) Human PBMC were incubated in 24-well plates coated with varying concentrations of anti-CD3 in combination with the indicated antibodies and stained with anti-CD69FITC/CD4+CD8-PE or CTx-FITC/CD4+CD8-PE and analyzed for the surface expression of CD69 or GM1 in the live-gated T-cell fraction. (D) Quantitation of Figure 1C. Representative data from three independent experiments are shown. 
lipid raft (Figure $1 \mathrm{C}$ and $\mathrm{D}$ ). Together, these results indicate that CD99 acts as a co-stimulatory molecule that sensitizes cells for activation through CD3 ligation.

\section{CD99 co-ligation amplifies signal 1}

Co-stimulatory molecules in $T$ cells can be categorized according to whether they amplify the generation of signal 1 or signal 2 (adhesion versus co-stimulation). In addition, the level of TCR downregulation reflects the number of functionally triggered TCRs and thus the amount of signal 1 (Valitutti et al., 1995). To test whether CD99 ligation plays a role in generating signal 1 , the level of TCR downregulation after co-ligation of CD3 and CD99 was examined. Interestingly, TCR stimulation with antiCD3+CD99 resulted in a two- to four-fold greater down-regulation of TCR than obtained with anti-CD3 alone or anti-CD3 + CD28 (Figure 2A). The diffe- rence in TCR down-regulation was most obvious at a low concentration of anti-CD3 (Figure 2B). This was also the case in a Jurkat T-cell line (Figure 2C).

\section{TCR/CD3 and CD99 co-engagement enhances tyrosine phosphorylation of the TCR $\zeta$-chain}

The ability of CD99 co-ligation to increase cell proliferation and accentuate signal 1 suggests that CD99 affects TCR signal strength by increasing the efficiency of the earliest signaling events generated upon TCR engagement. This hypothesis was tested by treating Jurkat $T$ cells with anti-CD99 plus antiCD3 and then comparing the extent of TCR $\zeta$ phosphorylation. Co-ligation of CD99 with suboptimal CD3 induced tyrosine phosphorylation of TCR $\zeta$ as did cells triggered by optimal engagement of CD3 (Figure $3 \mathrm{~A}$ ). The ratio of $\mathrm{p}-\mathrm{TCR} \zeta$ to total TCR $\zeta$ after CD99 co-ligation with suboptimal CD3 were approximately three- to four-fold higher than those ob-
A

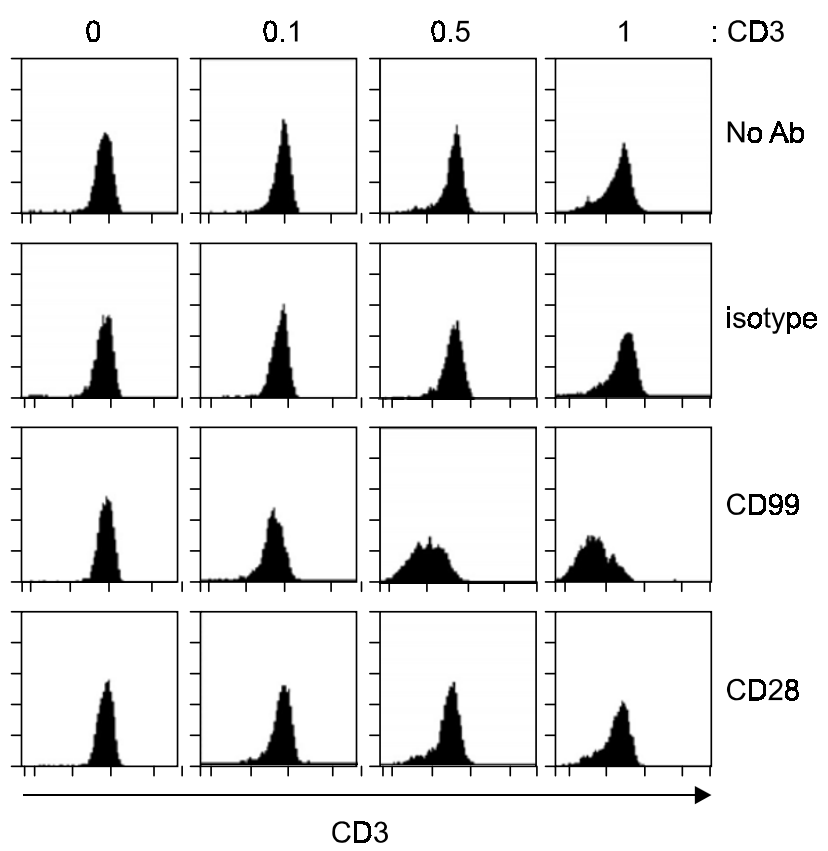

B
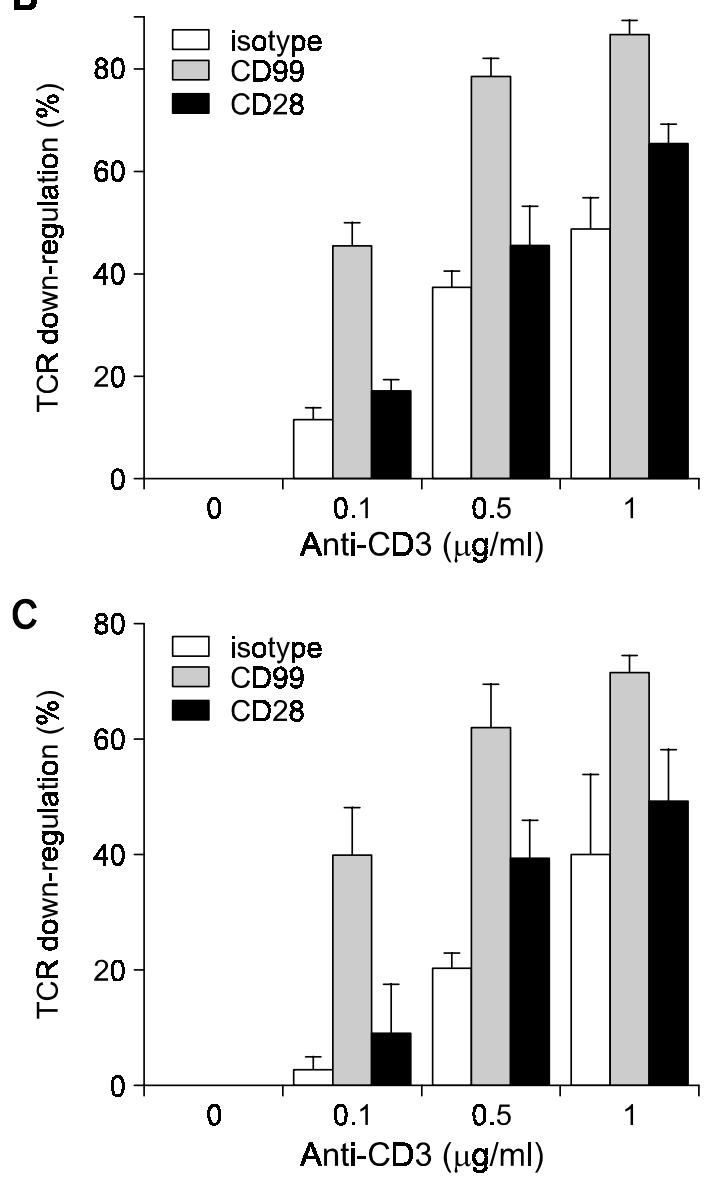

Figure 2. Enhanced TCR down-regulation after stimulation with anti-CD3 plus CD99. Human PBMC $(A, B)$ or Jurkat cells $(C)$ were treated as in Figure 1, and TCR expression was assessed $4 \mathrm{~h}$ later by flow cytometry. TCR down-regulation (\%) was calculated as a normalized value as follows: $100 \times($ MFI of TCR in resting cells - MFI of TCR in stimulated cells)/MFI of TCR in resting cells (B and C). MFI, Mean fluorescence intensity. 
served after cell treatment with suboptimal doses of anti-CD3 only (Figure 3B).

To exclude any possible artificial effect of antibody ligation, the same study was repeated with TCR$V \beta 3$-transfected Jurkat cells after stimulation with the superantigen SEB. V $\beta 3$-expressing Jurkat cells express two different TCR sets: transfected $\mathrm{V} \beta 3$ and the endogenously expressed V $\beta 8$ family. When V $\beta 3$ expressing Jurkat cells were stimulated with soluble

A

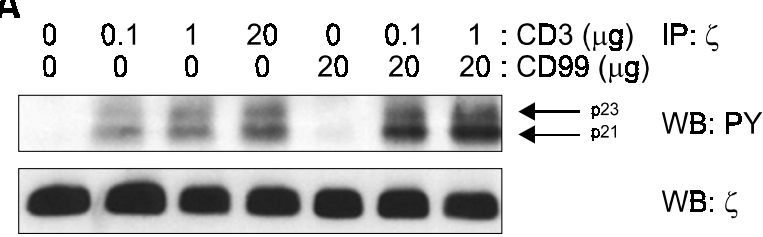

B

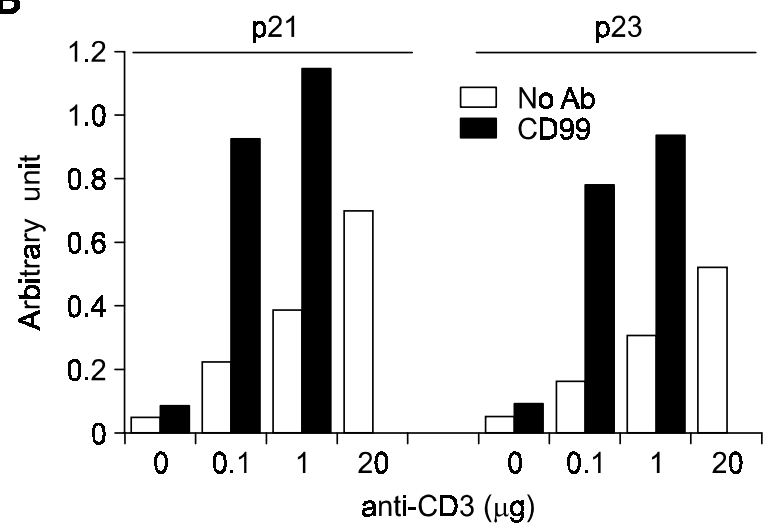

C

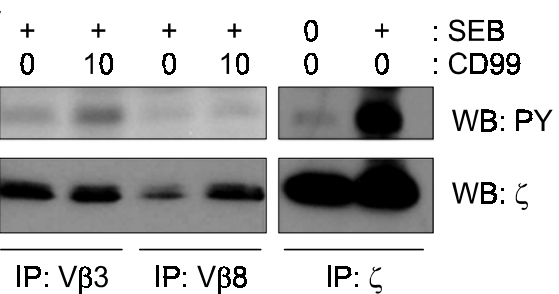

Figure 3. Enhanced phosphorylation of TCR $\zeta$ in cells co-stimulated by TCR and CD99 engagement. Jurkat T cells were stimulated for 5 min with varying concentrations of anti-CD3 mAb with or without anti-CD99 mAb. (A) Cells were subsequently lysed and immunoprecipitated with anti- $\zeta \mathrm{mAb}$. The precipitates were immunoblotted with anti-phosphotyrosine (4G10) mAb (top panel), stripped, and re-probed with anti- $\zeta \mathrm{mAb}$ as a loading control (bottom panel). (B) Densitometry of the phosphorylated (p23, p21) vs. non-phosphorylated -chains. (C) Jurkat cells expressing both V $B 3$ and $V \beta 8$ were activated with soluble SEB plus anti-CD99 and treated as above except that immunoprecipitations were done with anti-V $\beta$ mAbs. Representative data from three independent experiments are shown. PY, Phosphotyrosine; IP, immunoprecipitation; WB, Western blotting.
SEB in the presence of CD99 engagement, the level of $\zeta$-chain phosphorylation of $V \beta 3$ was greater than in cells stimulated with soluble SEB only. In contrast to $V \beta 3-\zeta$, the tyrosine residues on the $V \beta 8-\zeta$ were not phosphorylated (Figure $3 \mathrm{C}$ ). These data show that CD99-induced co-stimulation is specific for TCR engaged with SEB or anti-CD3 mAb.

\section{CD99 is weakly associated with the raft on resting and activated $\mathrm{T}$ cells}

Several studies have reported that some proteins residing in the raft fraction, such as CD5 and CD9, exhibit a co-stimulatory capacity by enhancing TCR signaling. To test whether CD99 belongs to these raft-resident co-stimulators, cell lysates of Jurkat $T$

A $1 \%$ Triton $\mathrm{X}-100$ lysis

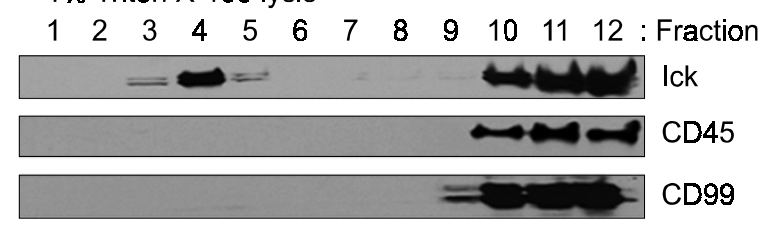

$1 \%$ Brij 58 lysis
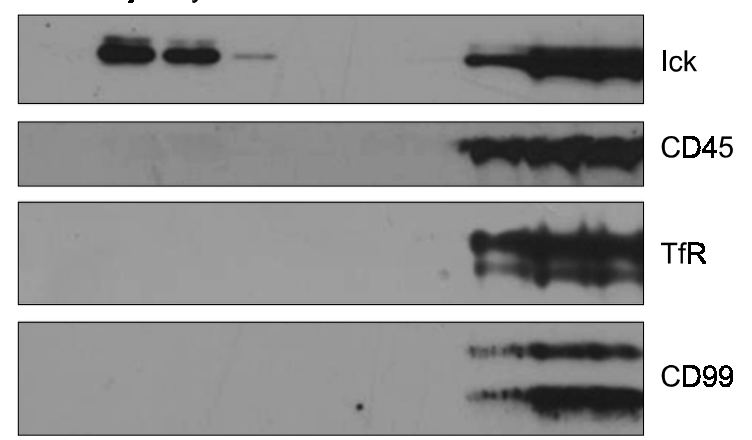

CD99

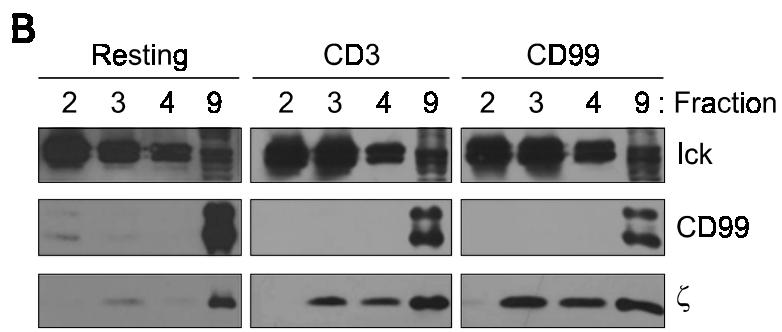

Figure 4. Distribution pattern of CD99 in resting and activated T cells. (A) Jurkat cells were treated with 1\% Triton X-100 (top) or Brij 58 lysis buffer (bottom) for 30 min on ice and then subjected to sucrose density gradient ultracentrifugation. Fractions were collected and equivalent portions of each fraction were analyzed by immunoblotting with the corresponding mAbs. (B) T cells were stimulated with irrelevant control $\mathrm{mAb}$, anti-CD3 or anti-CD99, and lysed with $1 \%$ Brij 58 lysis buffer. Protein bands were analyzed by probing with corresponding Abs. Representative data from three independent experiments are shown. 
cells were fractionated and the presence of CD99 in the raft fractions was checked by immunoblot analysis. We could detect CD99 marginally in lipid rafts of either resting or activated $T$ cells, which suggests none of stimulation conditions was able to coax the transport of CD99 molecules into raft fractions at a readily detectable level (Figure 4). The fragile association of CD99 molecules in the lipid raft clearly distinguishes CD99 from other raft-resident co-stimulatory molecules.

\section{CD99 engagement leads to translocation of the TCR complex into lipid rafts and facilitates TCR $\zeta$ phosphorylation in the presence of suboptimal CD3 stimulation}

The fact that CD99 engagement generates active phosphorylation of TCR $\zeta$ as well as aggregation of lipid rafts on the cell surface suggests that the CD99 signal augments the signal generated by suboptimal levels of CD3 ligation via transporting TCR into the raft area. Therefore, we examined the presence of TCR $\zeta$ and $\varepsilon$ chains in the raft fractions of cells stimulated with various combinations of anti-CD3 and CD99. Interestingly, the translocation of TCR $\zeta$ and $\varepsilon$ chains into the rafts was observed when CD99 ligation was included in the stimulation roster (Figures $4 \mathrm{~B}$ and $5 \mathrm{~A}$ ). The amounts of TCR $\zeta$ and $\varepsilon$ chains found in the raft fractions were almost similar to those in cells stimulated by optimal CD3 ligation or suboptimal CD3 plus CD99 co-ligation (Figure 5A and $B)$. In contrast, in the case of $C D 28$, neither CD28 single-ligation nor CD3+CD28 co-ligation induced translocation of TCR into lipid rafts.

To confirm the above data in intact cells, the positions of rafts and CD3 molecules in the sti-
A
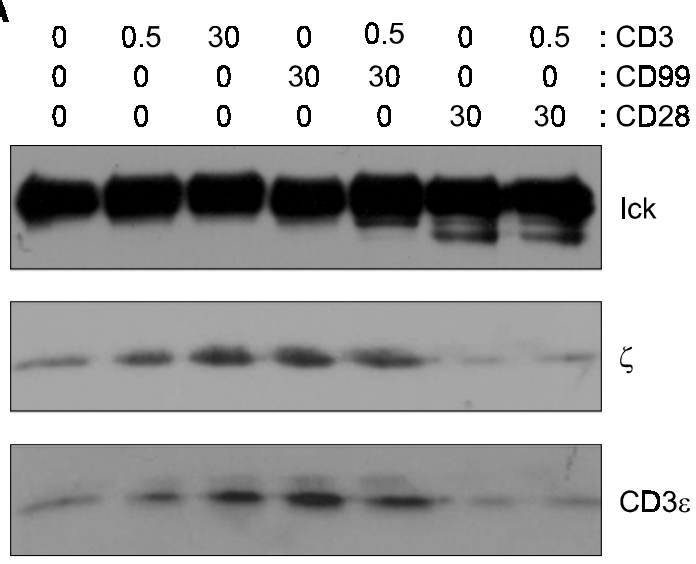

$\mathrm{CD} 3 \varepsilon$

C

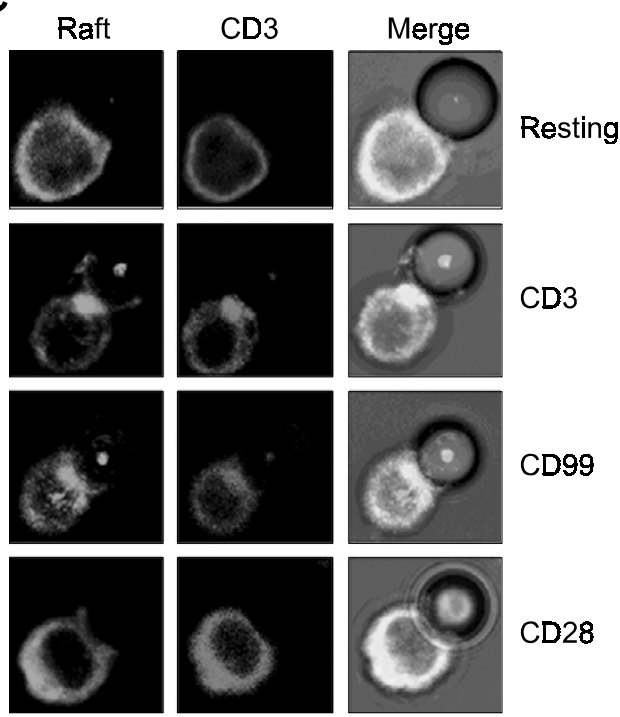

B

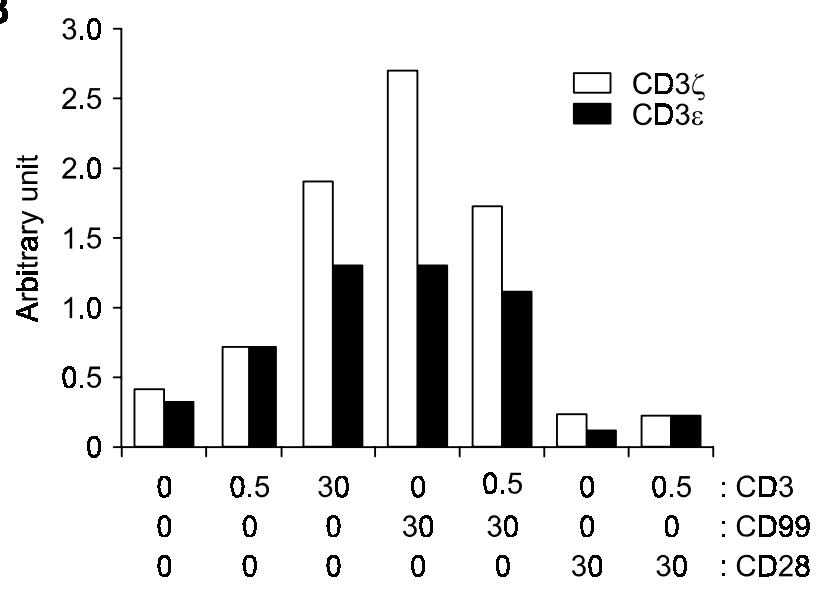

D

\begin{tabular}{|c|c|c|c|c|c|}
\hline 0 & 0.5 & 30 & 0 & 0.5 & : CD3 IP. $\zeta$ \\
\hline 0 & 0 & 0 & 30 & 30 & : CD99 \\
\hline neve & 4 & & & & WB: PY \\
\hline$=$ & $=$ & & & & WB: $\zeta$ \\
\hline
\end{tabular}

Figure 5. Translocation of TCR into the lipid raft upon CD99 engagement and accumulation of phosphorylated TCR $\zeta$ in the presence of CD3 ligation. (A) Jurkat cells were incubated with graded amounts of $\mathrm{mAb}$, as indicated at the top, and $1 / 40$ volumes of the pooled raft fractions were analyzed by immunoblotting with specific antibodies. (B) Densitometry of protein bands shown in A. (C) Jurkat cells were stained for confocal microscopy with CTX-FITC for lipid raft (green) and with anti-CD3-biotin followed by streptavidin-Texas Red for TCR/CD3 (red), after stimulation with mAbs-coated beads. (D) TCR $\zeta$ in the raft fractions was immunoprecipitated and probed with anti-PY mAb. Representative data from three independent experiments are shown. 
mulated cells were visualized by confocal microscopy. Consistent with the biochemical data, the engagement of cells with beads coated with antiCD99 $(100 \mathrm{mg} / \mathrm{ml})$ induced the redistribution and co-localization of CD3 molecules and rafts to the cell-to-bead contact sites, demonstrating that CD99 simulation indeed prompts the translocation of TCR $\zeta$ into lipid rafts (Figure $5 \mathrm{C}$ ).

To confirm whether the lipid raft indeed serves as the transduction site of the CD99 co-stimulatory function, the level of tyrosine phosphorylation on raft-bound TCR $\zeta$ was compared after stimulating cells with CD99 ligation alone or with co-ligation of CD99 and CD3. The presence of phosphorylated $\zeta$ chain was readily detected in cells given a suboptimal dose of anti-CD3 in conjunction with anti-CD99 (Figure 5D). Although a significant number of TCR $\zeta$ molecules were present in the rafts in the case of CD99 single-ligation, the extent of TCR $\zeta$ phosphorylation (assessed by the relative intensity of p-TCR $\zeta$ vs. that of total TCR $\zeta)$ under this condition was not much higher than in resting cells or in cells stimulated only with a suboptimal amount of anti- CD3. These results demonstrate that CD99 is capable of amplifying weak signals generated by suboptimal CD3 ligation to a level corresponding to that induced by optimal CD3 ligation, by increasing the local concentration of signaling molecules in the raft.

\section{Discussion}

We have demonstrated that the cell-surface protein CD99 acts as a co-stimulatory molecule in T-cell activation. The co-stimulatory function of CD99 was executed by enhancing the association of TCR $\zeta$ with lipid rafts. Upon additional stimulation with CD3, these raft-associated TCR complexes were extensively phosphorylated.

The degree of T-cell activation achieved by CD99 co-stimulation was comparable to that of CD28 (Figure 1). Previous reports have also shown the induction of IL-2 promoter activity by CD99 and CD28 (Waclavicek et al., 1998; Zhou et al., 2002). However, the inability of CD28 to affect TCR signal 1 and to translocate TCR $\zeta$ chains into rafts (Figures 2 and 5) clearly distinguishes the controlling mechanism of CD99 in the immune response from that of CD28. Furthermore, co-stimulation of CD28 differentiates naïve $T$ cells into the Th2 subtype (Shahinian et al., 1993; Skapenko et al., 2001), whereas CD99 co-stimulation induces the production of Th1-type cytokines in polyclonally activated T-cell lines (Waclavicek et al., 1998). These data suggest that CD99 provides a signal different from that of CD28, supporting previous notions that CD28 signaling is not related directly to TCR-derived signals (Reinhold et al., 1997; Alegre et al., 2001; Michel et al., 2001; Frauwirth and Thompson, 2002).

Other non-CD28 co-stimulatory molecules such as CD2 (Bierer et al., 1988), CD5 (Ledbetter et al., 1986), CD9 (Tai et al., 1996), and CD44 (Huet et al., 1989), also have a co-stimulatory effect on resting $T$ cells as potently as that of CD28 in the presence of suboptimal doses of anti-CD3 mAb. Co-ligation of these molecules and CD3 leads to increased $\mathrm{Ca}^{2+}$ mobilization, association of TCR/CD3 with rafts, and increased phosphorylation of LAT, compared to single ligation of CD3 (Yashiro-Ohtani et al., 2000). Yet, a major difference between these molecules and CD99 is their residency in the rafts of resting $T$ cells or of cells engaged with CD2 ligand (Yang and Reinherz, 2001; Badour et al., 2003). The fact that most CD99 molecules are far removed from the raft, and that even those few residing inside the raft maintain only a weak and fragile association under any circumstance (Figure 4), draws attention to how CD99 is able to translocate TCR $\zeta$ and $\varepsilon$ chains into rafts and increase TCR signaling. Equally intriguing is the identity of signal pathways involved in transducing CD99 stimulation. We checked Ick activity to test a hypothesis that CD3 plus CD99 stimulation enhances raft-associated kinase activity directly. However, we did not observe the differences between CD3 plus CD99 and CD3 alone (data not shown).

Evidence for a close relationship between CD99 and the cytoskeleton has accumulated, based on the function of CD99 as an adhesion molecule that induces homotypic cell aggregation (Bernard et al., 1995). CD99 engagement increases intracellular transport of TCR and MHC molecules to the cell surface in thymocytes (Choi et al., 1998) and elicits the exocytic transport of GM1 in Jurkat cells, accompanied by the rearrangement of actin cytoskeletons (Yoon et al., 2003). Furthermore, CD99 has been linked to the migration of monocytes (Schenkel et al., 2002) and to morphological changes in $T$ and $B$ cells (Kim et al., 1998; Bernard et al., 2000). Thus, it is highly probable that CD99-induced co-stimulation is mediated by cytoskeletal remodeling rather than enhancing TCR signaling directly.

In conclusion, CD99 was shown to function as a co-stimulatory molecule that is able to compensate for suboptimal CD3 ligation in promoting full activation of $\mathrm{T}$ cells; thereby amplifying signal 1 . It will be highly relevant to identify potential adaptor molecules acting downstream of CD99, not only to elucidate the exact mechanism of CD99 co-stimulation, but also to define functional and mechanistic differences between CD99 and other co-stimulatory molecules. 


\section{Acknowledgement}

We thank Sang Soon Yoon for the help with confocal microscopy; S. Chikuma and J. A. Bluestone for critical advices on cell stimulation and raft isolation. This work was supported by the 2003 National Research Laboratory fund (M1010400012401J0000 0551) from the Ministry of Science and Technology.

\section{References}

Alegre ML, Frauwirth KA, Thompson CB. T-cell regulation by CD28 and CTLA-4. Nat Rev Immunol 2001;1:220-8

Badour K, Zhang J, Shi F, McGavin MK, Rampersad V, Hardy LA, Field D, Siminovitch KA. The Wiskott-Aldrich syndrome protein acts downstream of CD2 and the CD2AP and PSTPIP1 adaptors to promote formation of the immunological synapse. Immunity 2003;18:141-54

Bernard G, Zoccola D, Deckert M, Breittmayer JP, Aussel C, Bernard A. The E2 molecule (CD99) specifically triggers homotypic aggregation of $\mathrm{CD} 4^{+} \mathrm{CD} 8^{+}$thymocytes. J Immunol 1995;154:26-32

Bernard G, Breittmayer JP, de Matteis M, Trampont P, Hofman $P$, Senik A, Bernard A. Apoptosis of immature thymocytes mediated by E2/CD99. J Immunol 1997;158:2543-50

Bernard G, Raimondi V, Alberti I, Pourtein M, Widjenes J, Ticchioni M, Bernard A. CD99 (E2) up-regulates alpha4beta1-dependent $T$ cell adhesion to inflamed vascular endothelium under flow conditions. Eur J Immunol 2000;30: 3061-5

Bierer BE, Peterson A, Gorga JC, Herrmann SH, Burakoff SJ. Synergistic $T$ cell activation via the physiological ligands for CD2 and the T cell receptor. J Exp Med 1988;168:1145-56

Bromley SK, Burack WR, Johnson KG, Somersalo K, Sims TN, Sumen C, Davis MM, Shaw AS, Allen PM, Dustin ML. The immunological synapse. Annu Rev Immunol 2001;19:375-96

Cerisano V, Aalto Y, Perdichizzi S, Bernard G, Manara MC, Benini S, Cenacchi G, Preda P, Lattanzi G, Nagy B, Knuutila S, Colombo MP, Bernard A, Picci P, Scotlandi K. Molecular mechanisms of CD99-induced caspase-independent cell death and cell-cell adhesion in Ewing's sarcoma cells: actin and zyxin as key intracellular mediators. Oncogene 2004;23: 5664-74

Cherukuri A, Dykstra M, Pierce SK. Floating the raft hypothesis: lipid rafts play a role in immune cell activation. Immunity 2001;14:657-60

Choi EY, Park WS, Jung KC, Kim SH, Kim YY, Lee WJ, Park $\mathrm{SH}$. Engagement of CD99 induces up-regulation of TCR and MHC class I and II molecules on the surface of human thymocytes. J Immunol 1998;161:749-54

Denning SM, Dustin ML, Springer TA, Singer KH, Haynes BF. Purified lymphocyte function-associated antigen-3 (LFA-3) activates human thymocytes via the CD2 pathway. J Immunol 1988; 141:2980-5

Frauwirth KA, Thompson CB. Activation and inhibition of lymphocytes by costimulation. J Clin Invest 2002;109:295-9
Frauwirth KA, Riley JL, Harris MH, Parry RV, Rathmell JC, Plas $\mathrm{DR}$, Elstrom RL, June $\mathrm{CH}$, Thompson $\mathrm{CB}$. The CD28 signaling pathway regulates glucose metabolism. Immunity 2002;16: 769-77

Green JM, Zheng XG, Shimizu Y, Thompson CB, Turka LA. T cell receptor stimulation, but not CD28 costimulation, is dependent on LFA-1-mediated events. Eur J Immunol 1994; 24:265-72

Huet S, Groux H, Caillou B, Valentin H, Prieur AM, Bernard A. CD44 contributes to T cell activation. J Immunol 1989;143: 798-801

Janes PW, Ley SC, Magee Al. Aggregation of lipid rafts accompanies signaling via the $\mathrm{T}$ cell antigen receptor. J Cell Biol 1999;147:447-61

Jang M, Lee M, Park H, Bae Y, Min DS, Ryu SH, Kwak J. Phosphorylation of phospholipase D1 and the modulation of its interaction with RhoA by cAMP-dependent protein kinase. Exp Mol Med 2004;36:172-8

Kim A, Noh Y, Kim KD, Jang Y, Choe Y, Lim J. Activated natural killer cell-mediated immunity is required for the inhibition of tumor metastasis by dendritic cell vaccination. Exp Mol Med 2004;36:428-43

Kim SH, Choi EY, Shin YK, Kim TJ, Chung DH, Chang SI, Kim NK, Park SH. Generation of cells with Hodgkin's and ReedSternberg phenotype through downregulation of CD99 (Mic2) Blood 1998;92:4287-95

Ledbetter JA, Parsons M, Martin PJ, Hansen JA, Rabinovitch $\mathrm{PS}$, June $\mathrm{CH}$. Antibody binding to CD5 (Tp67) and Tp44 T cell surface molecules: effects on cyclic nucleotides, cytoplasmic free calcium, and cAMP-mediated suppression. J Immunol 1986;137:3299-305

Ledbetter JA, Rabinovitch PS, Hellstrom I, Hellstrom KE, Grosmaire LS, June CH. Role of CD2 cross-linking in cytoplasmic calcium responses and $\mathrm{T}$ cell activation. Eur $\mathrm{J}$ Immunol 1988;18:1601-8

Michel F, Attal-Bonnefoy G, Mangino G, Mise-Omata S, Acuto O. CD28 as a molecular amplifier extending TCR ligation and signaling capabilities. Immunity 2001;15:935-45

Montixi C, Langlet C, Bernard AM, Thimonier J, Dubois C, Wurbel MA, Chauvin JP, Pierres MM, He HT. Engagement of T cell receptor triggers its recruitment to low-density detergentinsoluble membrane domains. EMBO J 1998;17:5334-48

Moran M, Miceli MC. Engagement of GPI-linked CD48 contributes to TCR signals and cytoskeletal reorganization: a role for lipid rafts in T cell activation. Immunity 1998;9:787-96

Reinhold MI, Lindberg FP, Kersh GJ, Allen PM, Brown EJ. Costimulation of $T$ cell activation by integrin-associated protein (CD47) is an adhesion-dependent, CD28-independent signaling pathway. J Exp Med 1997;185:1-11

Schenkel AR, Mamdouh Z, Chen X, Liebman RM, Muller WA. CD99 plays a major role in the migration of monocytes through endothelial junctions. Nat Immunol 2002;3:143-50

Shahinian A, Pfeffer K, Lee KP, Kundig TM, Kishihara KK, Wakeham A, Kawai K, Ohashi PS, Thompson CB, Mak TW. Differential $T$ cell costimulatory requirements in CD28-de- 
ficient mice. Science 1993;261:609-12

Skapenko A, Lipsky PE, Kraetsch HG, Kalden JR, SchulzeKoops $\mathrm{H}$. Antigen-independent Th2 cell differentiation by stimulation of CD28: regulation via IL-4 gene expression and mitogen-activated protein kinase activation. J Immunol 2001; 166:4283-92

Stillwell $R$, Bierer BE. T cell signal transduction and the role of CD7 in costimulation. Immunol Res 2001;24:31-52

Tai XG, Yashiro Y, Abe R, Toyooka K, Wood CR, Morris J, Long A, Ono S, Kobayashi M, Hamaoka T, Neben S, Fujiwara H. A role for CD9 molecules in $\mathrm{T}$ cell activation. J Exp Med 1996;184:753-8

Valitutti S, Muller S, Cella M, Padovan E, Lanzavecchia A. Serial triggering of many T-cell receptors by a few peptideMHC complexes. Nature 1995;375:148-51

Waclavicek W, Majdic O, Stulnig T, Berger M, Sunder-Plassmann R, Zlabinger GJ, Baumruker T, Stockl J, Ebner C, Knapp W, Pickl WF. CD99 engagement on human peripheral blood T cells results in TCR/CD3-dependent cellular activation and allows for Th1-restricted cytokine production. J Immunol 1998;161:4671-8

Xavier R, Brennan T, Li Q, McCormack C, Seed B. Membrane compartmentation is required for efficient $T$ cell activation. Im- munity 1998;8:723-32

Yang H, Reinherz EL. Dynamic recruitment of human CD2 into lipid rafts. Linkage to T cell signal transduction. J Biol Chem 2001;276:18775-85

Yashiro-Ohtani Y, Zhou XY, Toyo-Oka K, Tai XG, Park CS, Hamaoka T, Abe R, Miyake K, Fujiwara H. Non-CD28 costimulatory molecules present in $T$ cell rafts induce $T$ cell costimulation by enhancing the association of TCR with rafts. $J$ Immunol 2000;164:1251-9

Yoon SS, Jung KI, Choi YL, Choi EY, Lee IS, Park SH, Kim TJ. Engagement of CD99 triggers the exocytic transport of ganglioside $\mathrm{GM} 1$ and the reorganization of actin cytoskeleton. FEBS Lett 2003;540:217-22

Zhang S, Park K, Oh S, Cho H, Cho H, Park J, Cho Y, Koo B, Chae I, Choi D, Kim H, Lee M. NF-kB decoy potentiates the effects of radiation on vascular smooth muscle cells by enhancing apoptosis. Exp Mol Med 2005;37:18-26

Zhou XT, Yashiro-Ohtani Y, Nakahira M, Park WR, Abe R, Hamaoka T, Naramura M, Gu H, Fujiwara H. Molecular mechanisms underlying differential contribution of CD28 versus non-CD28 costimulatory molecules to IL-2 promote activation. J Immunol 2002;168:3847-54 